RESEARCH REPORT

\title{
Patterns and severity of vincristine-induced peripheral neuropathy in children with acute lymphoblastic leukemia
}

\author{
Ellen M. Lavoie Smith ${ }^{1}$, Lang $\mathrm{Li}^{2}$, ChienWei Chiang ${ }^{2}$, Karin Thomas ${ }^{3}$, Raymond J. \\ Hutchinson ${ }^{1}$, Elizabeth M. Wells ${ }^{4}$, Richard H. Ho ${ }^{5}$, Jodi Skiles ${ }^{2}$, Arindom Chakraborty ${ }^{2}$, \\ Celia M. Bridges ${ }^{1}$, and Jamie Renbarger ${ }^{2}$ \\ ${ }^{1}$ School of Nursing, University of Michigan, Ann Arbor, MI, USA; ${ }^{2}$ School of Medicine, Indiana University, Indianapolis, IN, \\ USA; ${ }^{3}$ Department of Nursing, Children's Hospital Colorado, Aurora, CO, USA; ${ }^{4}$ Children's National Health System, \\ Washington, DC, USA; and ${ }^{5}$ Department of Pediatrics, Vanderbilt University, Nashville, TN, USA
}

\begin{abstract}
Vincristine, a critical component of combination chemotherapy treatment for pediatric acute lymphoblastic leukemia (ALL), can lead to vincristine-induced peripheral neuropathy (VIPN). Longitudinal VIPN assessments were obtained over 12 months from newly diagnosed children with ALL $(N=128)$ aged $1-18$ years who received vincristine at one of four academic children's hospitals. VIPN assessments were obtained using the Total Neuropathy Score-Pediatric Vincristine (TNS@-PV), National Cancer Institute Common Terminology Criteria for Adverse Events (CTCAE@), Balis@ grading scale, and Pediatric Neuropathic Pain Scale@-Five (PNPS@-5). Of children who provided a full TNS@-PV score, 85/109 (78\%) developed VIPN (TNS@-PV $\geq 4$ ). Mean TNS@-PV, grading scale, and pain scores were low. CTCAE@-derived grades 3 and 4 sensory and motor VIPN occurred in $1.6 \% / 0 \%$, and $1.9 \% / 0 \%$ of subjects, respectively. VIPN did not resolve in months 8-12 despite decreasing dose density. VIPN was worse in older children. Partition cluster analysis revealed 2-3 patient clusters; one cluster $(n=14)$ experienced severe VIPN. In this population, VIPN occurs more commonly than previous research suggests, persists throughout the first year of treatment, and can be severe.
\end{abstract}

Key words: acute lymphoblastic leukemia, children, patterns, severity, vincristine-induced peripheral neuropathy

\section{Introduction}

Acute lymphoblastic leukemia (ALL), the most common childhood cancer, was diagnosed in 2,670 children and 410 adolescents in the United States in 2014 (American Cancer Society, 2014). Because of advances in treatment, including the use of vincristine

Address correspondence to: Ellen M. Lavoie Smith, PhD, APN-BC, AOCN, School of Nursing, University of Michigan, $400 \mathrm{~N}$ Ingalls, Ann Arbor, MI 48109, USA. Tel: +(734)936-1267; Fax +(734)936-5525;

E-mail: ellenls@med.umich.edu as a mainstay chemotherapeutic agent, the 5-year survival rate increased from $57 \%$ in $1975-1979$ to $90 \%$ in 2003-2009 (American Cancer Society, 2014). Many children who undergo vincristine treatment experience vincristine-induced peripheral neuropathy (VIPN), which affects sensory, motor, and autonomic nerves (Toopchizadeh et al., 2009; Gomber et al., 2010; Anghelescu et al., 2011; Argyriou et al., 2012). Moderate to severe VIPN (grades 3 and 4) necessitates decreasing the vincristine dose (Verstappen et al., 2005; Gomber et al., 2010), which may affect cancer treatment efficacy. VIPN may not resolve over time, which negatively 
influences function and quality of life for years beyond treatment completion (Postma et al., 1993; Ness et al., 2013).

Although VIPN is recognized as a common adverse effect of pediatric ALL treatment, little is known about its true incidence, severity, clinical manifestations, and patterns experienced over the first year of therapy, due in part to the difficulty in accurately capturing neuropathy in young children. In pediatric studies of vincristine-based treatments for ALL, sarcoma, and medulloblastoma, most authors report low National Cancer Institute (NCl) Common Terminology Criteria (CTCAE@)-derived grades 3 and 4 VIPN incidence rates ranging from $0 \%$ to $37 \%$ (Vats et al., 1992; Kortmann et al., 2000; Chauvenet et al., 2003; Bisogno et al., 2005; Ramchandren et al., 2009; Messinger et al., 2010; Messinger et al., 2012). The highest incidence was reported when vincristine was administered with other neurotoxic drugs (Kortmann et al., 2000). Incidence rates were also higher in studies reporting all levels of VIPN severity, as opposed to just severe (grades 3 and 4) VIPN, and when more rigorous VIPN assessment approaches were used, that is, clinical examinations and electrophysiological studies (Verstappen et al., 2005; Ramchandren et al., 2009; Toopchizadeh et al., 2009). For example, rigorous VIPN assessment revealed that nearly all participants $(96 \%$ of $n=25)$ developed VIPN (Toopchizadeh et al., 2009). Grading scales, such as the CTCAE@, have been criticized for being insensitive and unreliable (Postma et al., 1998; Cavaletti et al., 2010; Griffith et al., 2010; Frigeni et al., 2011; Cavaletti et al., 2013; Gilchrist et al., 2014). When considering the high VIPN incidence reported in studies using rigorous assessment approaches as opposed to the low VIPN incidence rates reported in studies using the CTCAEC, it is clear that reliance on grading scales to quantify VIPN in clinical trials has led to false impressions about the scope of the problem.

Although young children are unable to describe their VIPN symptoms (Gilchrist, 2012), clinicians often rely on childrens' self-report (Paice, 2009; Cavaletti et al., 2010). Worsening neurotoxicity and appropriate vincristine dose adjustments may be neglected, leading to worsening or permanent nerve damage.

Chronic, even if subtle, peripheral neuropathy over a childhood cancer survivor's lifetime may be significant. For example, in a study of long-term survivors treated for pediatric malignancies, patients with vincristine- or platinum-associated peripheral neuropathy experienced chronic sensory impairment (Ness et al., 2013). Motor deficits may result in decreased physical activity, obesity, type 2 diabetes mellitus, metabolic syndrome, and cardiovascular disease (Hoffman et al., 2013; Khan et al., 2014). Understanding VIPN and identifying the highest risk children could facilitate optimizing vincristine dosing for maximum disease response while minimizing the risk of chronic functional deficits.

We evaluated VIPN throughout treatment using reliable, valid, sensitive, and responsive assessment approaches to quantify the VIPN phenotype. Study aims were to describe (1) VIPN incidence, patterns, and severity; (2) the relationship between dose density and VIPN; and (3) predictors of more severe VIPN when assessed over the first 12 months of vincristine therapy in children ages 1-18 with ALL.

\section{Methods}

\section{Sample and setting}

Children with newly diagnosed precursor B-cell ALL $(N=128)$ were recruited from four academic medical centers: Indiana University School of Medicine/Riley Hospital for Children, the University of Michigan Comprehensive Cancer Center/Mott Children's Hospital, Vanderbilt University/Monroe Carell Jr Children's Hospital, and George Washington University/Children's National Medical Center. Participants were between the ages of 1 and 18 at the time of diagnosis and received vincristine according to Children's Oncology Group (COG) treatment trials (including AALL0232, AALL0331, AALL08P1, or AALL0932). The standard vincristine dosage received was $1.5 \mathrm{mg} / \mathrm{m}^{2}$ (capped at 2-mg maximum dose). Toxicity-based dose modifications were defined according to the specific COG protocol guiding the individual child's leukemia treatment. The exclusion criteria were (1) baseline peripheral neuropathy score greater than grade 1 per the $\mathrm{NCl}$ CTCAE@ version 4.0; (2) currently receiving erythropoietin, itraconazole, or vitamin supplement greater than $100 \%$ of the recommended daily allowance; (3) Down syndrome; (4) pregnancy; and (5) a history of coexisting serious illness that would limit neurological assessments.

\section{Measures}

VIPN was assessed using the Total Neuropathy Score (TNS@), NCI CTCAE@ V.4.0, and the Modified Balis Pediatric Scale of Peripheral Neuropathy@ (Smith et al., 2008). We used a TNS@ subscale that had been revised for use in children receiving vincristine (TNS@-PV) (Table 1) (Smith et al., 2013).

VIPN-associated pain was measured using the Pediatric Neuropathic Pain Scale@-Five (PNPS@-5). The FACES@ pain scale (Bosenberg et al., 2003; Hockenberry, 2005) was used to assist children to select a PNPSC-5 pain severity rating. If the child did not understand the question, parents/guardians estimated the pain scores based on observations of their children. 


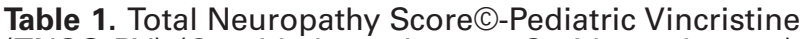
(TNS(C)-PV) (Cornblath, et al., 1999; Smith, et al., 2013).

\begin{tabular}{|c|c|}
\hline Item & Description \\
\hline $\begin{array}{l}\text { Worse subjective } \\
\text { symptom }\end{array}$ & $\begin{array}{l}\text { The worse score obtained based } \\
\text { on the distal to proximal } \\
\text { extension of paresthesias, } \\
\text { numbness, and neuropathic } \\
\text { pain }\end{array}$ \\
\hline Temperature sensibility & Test distal to proximal \\
\hline Vibration sensibility & Test distal to proximal \\
\hline Strength & $\begin{array}{l}\text { Toes, ankles, hips, hands, } \\
\text { thumbs, wrist, arm }\end{array}$ \\
\hline Deep tendon reflexes & $\begin{array}{l}\text { Ankle, knee, supinator, triceps, } \\
\text { biceps }\end{array}$ \\
\hline Autonomic neuropathy & Constipation \\
\hline Laryngeal neuropathy & Vocal cord function (hoarseness) \\
\hline
\end{tabular}

\section{Procedure}

The study was approved by each site's institutional review board. If the subjects were eligible, parent/guardian consent was obtained, along with the child's assent for children $\geq 7$ years of age.

VIPN assessments using the TNS@-PV were performed by trained evaluators (nurses, physicians, mid-level providers, and students) who had passed a competency examination judged by a pediatric neurologist. Neurologist-evaluator inter-rater reliability (IRR) was assessed at each site periodically throughout the study (methods reported elsewhere) (Smith et al., 2013). TNS@-PV score correlations between neurologists and non-neurologist raters were moderate to strong ( $r=0.54-0.99)$ and IRR was better when the alternative scoring approach was used (Smith et al., 2013).

Subjects were evaluated for neuropathy and pain at baseline (before day 8 vincristine) and, for the first year, on each subsequent day of vincristine treatment prior to vincristine administration.

\section{Analyses}

Only data from children who could provide complete TNS@-PV scores were included in the analyses. To assess for likelihood of selection bias, the demographic characteristics of children who could not provide a complete score were compared to the analyzed sample. Children with TNS@-PV score $\geq 4$ were defined as having VIPN, a cut-point based on findings that approximately $5 \%$ of pediatric normal controls have a ped-mTNS(C) (a slightly different TNS(C) variant) score of four or higher, and no normal controls had scores $\geq 5$ (Gilchrist and Tanner, 2013). Data were analyzed using descriptive statistics (frequencies, means, and standard deviations [SDs]), two-tailed correlations, paired $t$-tests, one-way ANOVA with Tukey's post hoc tests, and cluster analysis. Vincristine dose density curves were calculated based on the kernel density function. Finding patient groups showing similar patterns for each of the items was accomplished through a cluster analysis technique: the $k$-medoids or Partition Around Medoids (PAM) technique (Kaufman and Rousseeuw, 1987), which is a modification of the standard $k$-means clustering method. The only difference from $k$-means is that each cluster in PAM is represented by one object (middle object or median-like object) in the cluster instead of the cluster center. The goal of the algorithm is to minimize the average dissimilarity of objects to their closest selected object. We also minimized the sum of the dissimilarities between object and their closest selected object. Starting from an initial set of medoids, the PAM method replaced one of the medoids by a nonmedoid. When it improved the aggregate similarity measure, we retained the swap. This process was repeated for all medoid-nonmedoid pairs. For the similarity measure, we used Euclidian distances which are root sum of squares of differences. In our analysis, the pre-specified $k$ value was 3 for 12 months. Missing data were imputed using the average value of that month. Cluster analyses of TNS@-PV data were conducted using the function pam in R 3.0.2.

Patients received vincristine every week during the first 4 weeks of treatment, but less frequently during the maintenance period. To address this unbalanced dose-response relationship, we observed the vincristine treatment density over all time points, and transformed the dose time points into dose density. To transform discrete time points into a continuous time curve with the area under the curve equal to 1 , the dose density curve was computed using the density function in R 3.0.2., in which a greater magnitude of curve represents higher frequency dosing. Via teleconferences, a qualitative analytic approach was used to interpret the dose density curves and the relationships between dose density and VIPN severity.

\section{Results \\ Demographics}

A total of 1,539 assessments were performed on 128 children in their first year of treatment. Of those children, 109 were able to provide multiple complete scores over the first year, and 19 were never able to provide a complete score (excluded from most analyses). Table 2 presents sample demographic characteristics based on the total number of assessments performed in the first year. Using data from those providing complete TNSO-PV scores ( $N=109)$, there were nearly an equal number of assessments performed on males $(47 \%)$ and females (53\%) and most were performed on Caucasian (88\%) and non-Hispanic (78\%) 
subjects. The mean number of TNS@-PV assessments conducted on each child was $12.3(\mathrm{SD}=6.4)$. The mean age was 7.5 years, with a range at baseline of 1-19 years. When comparing the analyzed sample of children who provided full TNS@-PV scores $(n=109)$ to those children excluded from the analysis $(n=19)$, the children in the excluded cohort were significantly younger $(p<0.001)$ and more were males $(p<0.001)$.

\section{VIPN incidence, patterns, and severity}

Over the first year of treatment, $78 \%$ of the children who provided complete TNS@-PV scores developed VIPN. Mean total and item scores, ranges, and SDs are presented in Table 3. Mean TNS@-PV individual item scores were low for most children.

Mean sensory and motor CTCAE@ scores are also provided in Table 3. CTCAE@-derived grades 1, 2, 3, and 4 sensory VIPN occurred in $31 \%, 3.2 \%, 1.6 \%$, and $0 \%$, and motor VIPN in $18 \%, 4.4 \%, 1.9 \%$, and $0 \%$ of children, respectively. Balis scale grades were similar to the CTCAE grades. CTCAE $\bigcirc$ motor and sensory scores were $>0$ in $69 \%$ and $74 \%$ of the sample, respectively.

FACES(C) pain scores were attainable in nearly all children (96\%). Approximately 44\% reported VIPNassociated pain (FACESC score $>0$ ). Pain severity was mild in most cases (mean score $=0.19$; $S D=0.72$ ). PNPS@-5 item scores - attainable in fewer children $(83 \%)$ - suggest that foot pain, although mild, was slightly more severe than other pain types (mean/SD $=0.11 / 0.52$ ) (Table 3).

Figures $1 \mathrm{~A}$ and $1 \mathrm{~B}$ illustrate patterns of individual TNS@-PV item scores over the first 12 months of treatment. Reflexes were affected the most, followed by vibration sensibility and strength. Most item scores were highest at month 6 . Cranial neuropathy was minimal, worsened in month 2, and completely resolved by month 11.

The cluster analysis revealed three distinct VIPN severity subgroups (high, middle, and low) (Fig. 2). TNS@-PV scores in the high cluster/cluster 1 subgroup $(n=14)$ initially peaked 4 months from treatment onset, which was approximately 2 months after reaching the maximum vincristine dose density (time of peak vincristine administration frequency), illustrating a coasting effect. TNS@-PV scores for the middle cluster/cluster 2 subgroup $(n=70)$ peaked at month 2 and then remained essentially stable over the 12 month assessment period. Some patients $(n=25)$ fell into the low VIPN severity cluster/cluster 3 . These patients experienced minimal VIPN and their TNS@-PV scores spiked at months 2, 6, and 10. In all cluster subgroups, VIPN scores remain abnormal at the 12-month time point.
Figure 3 and Table 4 illustrate the variation in individual TNS@-PV items scores by cluster grouping. When compared to the other item scores and regardless of cluster grouping, tendon reflex scores were the highest (most abnormal), followed by diminished vibration sensation, and then altered strength. Cranial neuropathy occurred infrequently. Table 4 also shows that all pain item scores were higher in cluster 1 than in the other two clusters.

CTCAE@ grade cluster patterns were very similar to those of the TNS@-PV (Table 4). Regardless of measurement approach, three separate clusters emerged. Mean CTCAE@ scores by cluster suggest that sensory neuropathy was more severe than motor (Table 4). Additional analyses were conducted to assess for confounding variables that could have influenced the cluster analysis findings. We found differences in the cluster groupings based on the enrollment site. TNS@-PV scores were significantly higher in patients enrolled at the George Washington University/Children's National Medical Center than in patients enrolled at the other sites $(p=0.002)$. Fewer George Washington University patients fell into the low TNS@-PV and CTCAE@ clusters when compared to the other sites. Differences in VIPN scores by enrollment site could not be explained based on differences in age, gender, race, or pharmacokinetic parameters (vincristine clearance or area under the curve).

We conducted analyses to explore whether steroid-induced myopathy could have confounded the VIPN scores. A case-by-case analysis of all patients with high TNS@-PV motor scores was conducted to identify muscle weakness in patients with no other signs of VIPN. Because we found no cases of motor score weakness in the first 6-8 weeks of vincristine treatment (when steroid myopathy is most likely to occur) in the absence of other VIPN signs and symptoms, motor scores probably do not reflect steroid myopathy.

\section{Predictors of more severe VIPN}

TNS@-PV scores were positively associated with age $(r=0.31 ; p<0.0001)$ (Table 5). All individual TNS@-PV item scores were higher in older children except for the autonomic and cranial neuropathy items. CTCAE@ sensory and motor grades also were higher in older children. No significant differences in neuropathy scores based on race or gender were found.

TNS@-PV total and individual item scores did not significantly improve in months 8-12 despite decreasing vincristine dose density over the same time (Fig. 2). Some children $(n=14)$ experienced severe VIPN that appears to be unrelated to dose density (Fig. 2). 
Table 2. Sample demographics.

\begin{tabular}{|c|c|c|c|}
\hline & Total sample & $\begin{array}{l}\text { Analyzed sample with } \\
\text { Full TNS@C-PV Scores }\end{array}$ & Excluded sample \\
\hline Sample (N) & 128 & 109 & 19 \\
\hline Assessments (N) & 1,539 & 1,338 & 201 \\
\hline \multicolumn{4}{|l|}{ Age } \\
\hline Mean (SD) & $6.84(4.16)$ & $7.45(4.10)$ & $2.78(1.20)^{*}$ \\
\hline Range & $1-19$ & $1-19$ & $1-10$ \\
\hline \multicolumn{4}{|l|}{ Gender, n (\%) } \\
\hline Male & $777(50.5)$ & $631(47.2)$ & $146(72.6)^{*}$ \\
\hline Female & $763(49.5)$ & 707 (52.8) & $55(27.4)$ \\
\hline \multicolumn{4}{|l|}{ Race } \\
\hline Caucasian & 1,360 (88.3) & 1,177 (88.0) & $183(91.0)$ \\
\hline African American & $77(5.0)$ & $77(5.8)$ & $0(0.0)$ \\
\hline Asian & $4(0.3)$ & $4(0.3)$ & $0(0.0)$ \\
\hline Unknown & $98(6.4)$ & $80(6.0)$ & $18(9.0)$ \\
\hline \multicolumn{4}{|l|}{ Ethnicity } \\
\hline Hispanic & $186(12.1)$ & $144(10.8)$ & $42(20.9)$ \\
\hline Non-Hispanic & $1,178(76.5)$ & $1,042(77.9)$ & $136(67.7)$ \\
\hline Unknown & $175(11.4)$ & $152(11.4)$ & $23(11.4)$ \\
\hline \multicolumn{4}{|c|}{ Assessments per patient } \\
\hline Mean (SD) & $12.02(6.63)$ & $12.27(6.42)$ & $10.58(7.76)$ \\
\hline Range & $1-25$ & $1-25$ & $1-22$ \\
\hline
\end{tabular}

TNS@-PV, Total Neuropathy Score-Pediatric Vincristine.

* Significantly different from analyzed sample at $p<0.0001$ based on a two-tailed $t$-test.

Table 3. Mean VIPN and pain scores $(n=109)$.

\begin{tabular}{|c|c|c|c|c|c|}
\hline Scale and items & & $\begin{array}{l}\text { Assessments } \\
\text { obtained }\end{array}$ & Range & Mean & SD \\
\hline TNS@-PV items & $\mathrm{N}$ (Assessments) & $\%$ & & & \\
\hline Paresthesias & 1,259 & 94 & $0-4$ & 0.19 & 0.75 \\
\hline Numbness & 1,258 & 94 & $0-4$ & 0.13 & 0.62 \\
\hline Neuropathic pain & 1,264 & 94 & $0-4$ & 0.23 & 0.80 \\
\hline Temperature & 1,153 & 86 & $0-4$ & 0.32 & 0.81 \\
\hline Vibration & 1,118 & 84 & $0-4$ & 0.73 & 1.12 \\
\hline Strength & 1,224 & 91 & $0-4$ & 0.54 & 0.79 \\
\hline Reflex & 1,229 & 92 & $0-4$ & 1.52 & 1.30 \\
\hline Autonomic & 1,318 & 99 & $0-4$ & 0.52 & 0.79 \\
\hline Cranial & 1,318 & 99 & $0-2$ & 0.06 & 0.27 \\
\hline Total score & 1,066 & 80 & $0-22$ & 4.08 & 3.56 \\
\hline CTCAE $(\mathbb{C}$ sensory & 1,133 & 85 & $0-3$ & 0.34 & 0.62 \\
\hline CTCAE@ motor & 1,133 & 85 & $0-3$ & 0.43 & 0.61 \\
\hline FACES $\subset$ & 1,286 & 96 & $0-5$ & 0.19 & 0.72 \\
\hline \multicolumn{6}{|l|}{ NPS(C-CIN items } \\
\hline Feet pain & 1,110 & 83 & $0-5$ & 0.10 & 0.52 \\
\hline Hand pain & 1,111 & 83 & $0-4$ & 0.04 & 0.30 \\
\hline Jaw pain & 1,110 & 83 & $0-5$ & 0.04 & 0.26 \\
\hline Numb pain & 1,105 & 83 & $0-5$ & 0.09 & 0.46 \\
\hline Tingly pain & 1,103 & 82 & $0-5$ & 0.09 & 0.47 \\
\hline
\end{tabular}

CTCAE $\subseteq$, Common Terminology Criteria; TNS $\odot-P V$, Total Neuropathy Score-Pediatric Vincristine; VIPN, vincristine-induced peripheral neuropathy.

\section{Discussion}

Our use of the sensitive TNS@-PV assessment tool revealed that $78 \%$ of children developed VIPN and $44 \%$ reported pain; however, symptom severity was generally low. Although the incidence rates we found are discordant with other published reports, we believe that our findings are valid because we used rigorous and validated methods for quantifying the VIPN phenotype. The large sample size (1,338 assessments in 109 children), longitudinal study design (12 months), and use of TNS@-PV allowed more accurate VIPN characterization than that of previously published studies using smaller samples and retrospective or cross-sectional designs. These factors may explain the higher VIPN incidence 
A
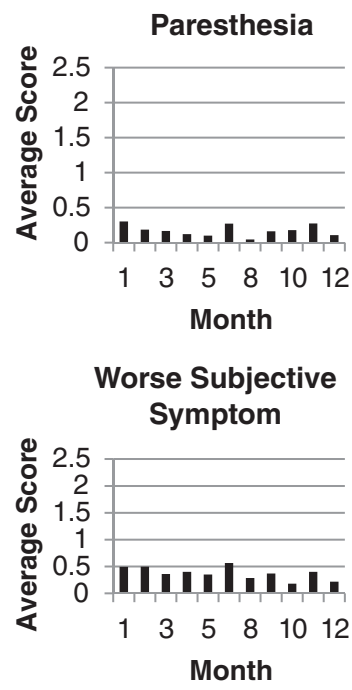

B

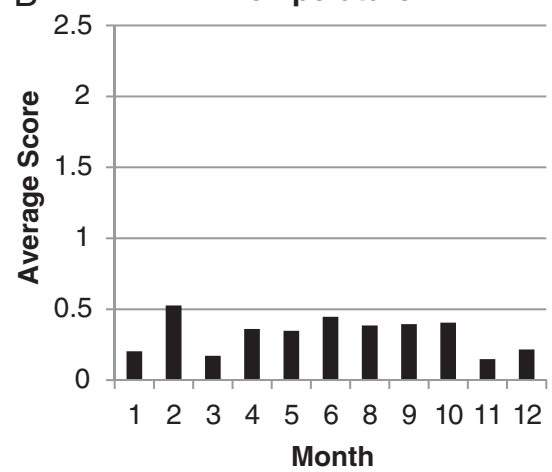

Strength

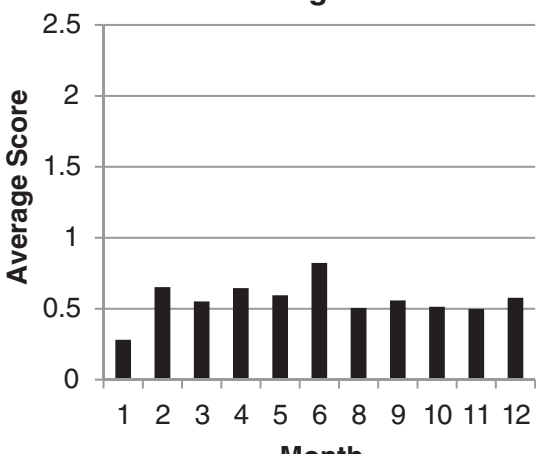

Numbness

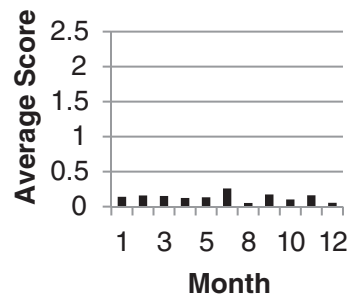

AutonomicConstipation

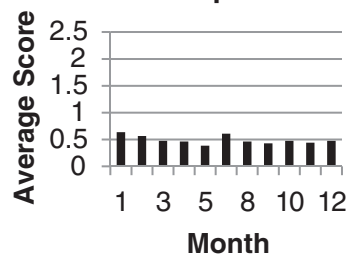

Neuropathic Pain

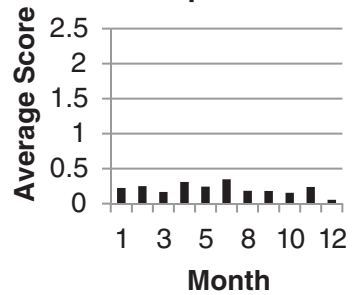

Cranial-

Hoarseness

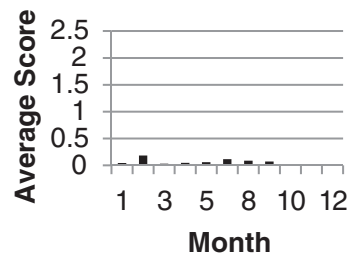

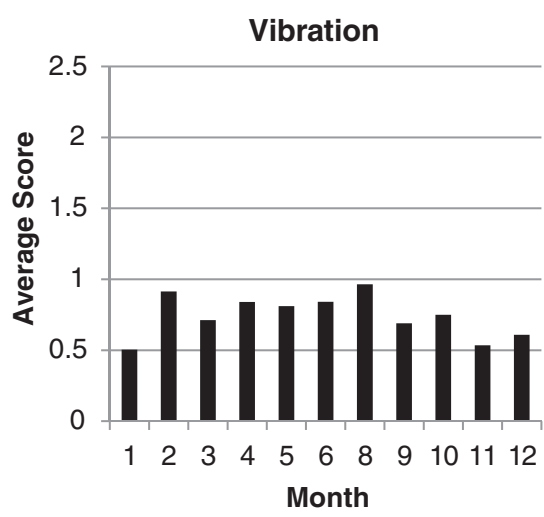

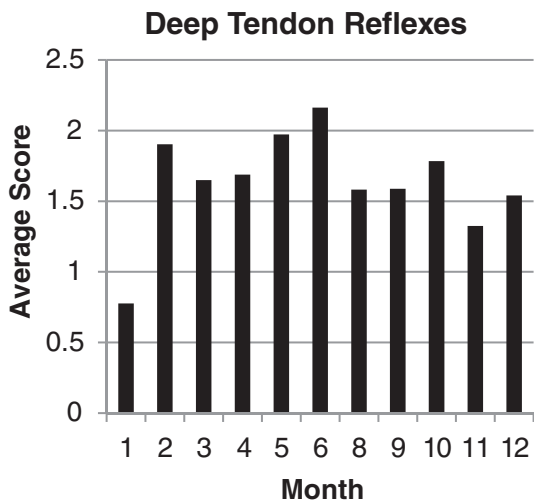

Figure 1. (A) Total Neuropathy Score-Pediatric Vincristine (TNS@-PV) Subjective Symptom Scores in first 12 months $(n=109)$. (B) TNS@-PV Objective Sign Scores in first 12 months $(n=109)$.

rates discovered in this study. When comparing VIPN rates identified using the TNS@-PV to the traditional $\mathrm{NCI}$ CTCAE@, the TNS-PV identified slightly more children with VIPN. Our findings are similar (but less striking) than results reported by Gilchrist et al., who discovered that the CTCAE@ failed to uncover VIPN in $40 \%$ of children $5-18$ years of age with ped-mTNSC scores $\geq 5$ (Gilchrist et al., 2014). It is highly likely that future use of a TNS@-based assessment approach will lead to higher and more accurate VIPN incidence rates than have previously been reported based on the CTCAE@.

VIPN did not improve despite children receiving less vincristine in months 8-12. Additional research is ongoing to assess long-term outcomes in this cohort beyond the 12 month time point. 


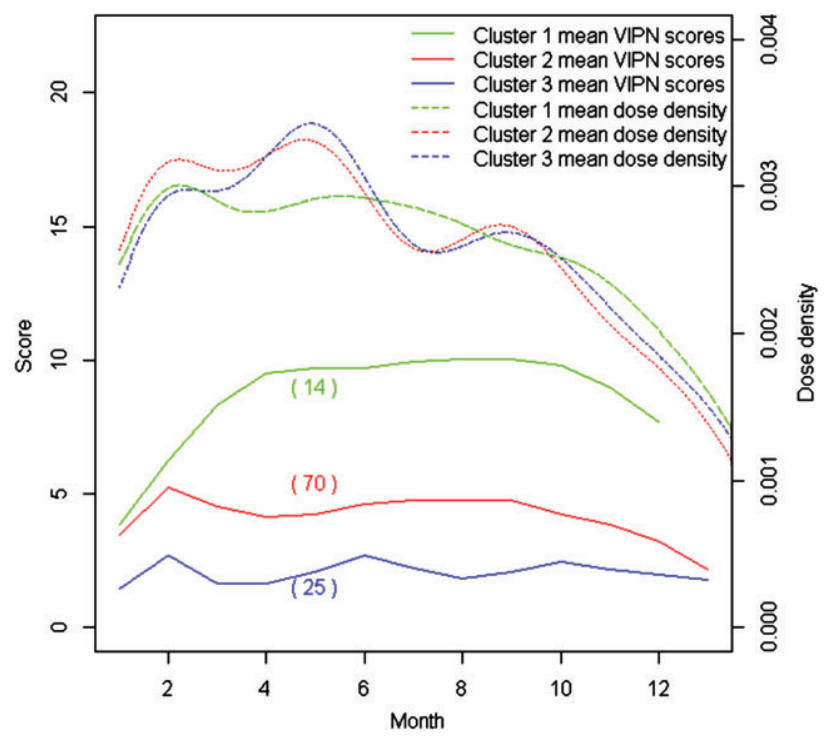

Figure 2. Total Neuropathy Score-Pediatric Vincristine (TNS@-PV) Mean Total Score and dose density by cluster $(n=109)$.

VIPN scores were worse in older children. Our results may partly relate to the enhanced ability of older children to describe their symptoms, whereas younger children may lack the vocabulary or the understanding to do so. Our findings are consistent with results published by Liew et al. showing that VIPN severity in adults with ALL was also worse in older patients $(p=0.0006)$ (Liew et al., 2013). Our findings are also

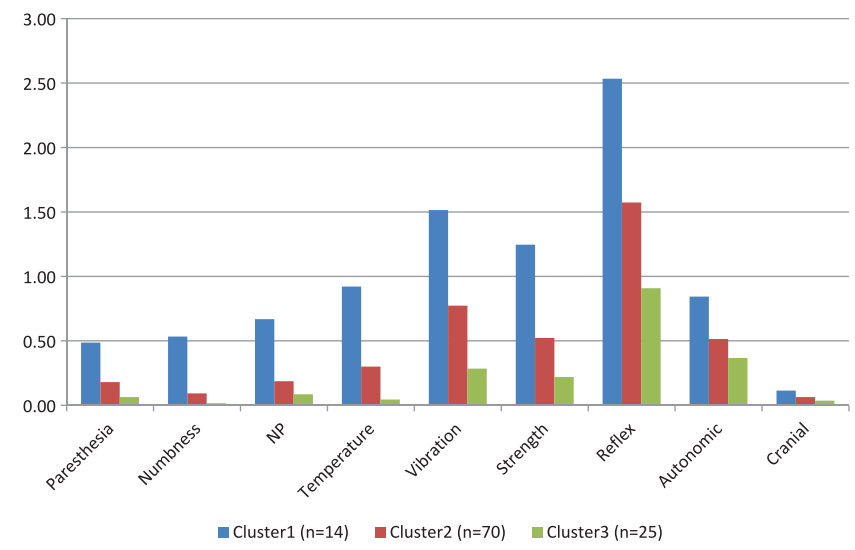

Figure 3. Mean Total Neuropathy Score-Pediatric Vincristine (TNS@-PV) Item Scores by cluster $(n=109)$.

consistent with the adult ALL literature and seem to confirm anecdotal clinical observations that older children experience worse VIPN. In order to validate our findings further, more accurate and precise methods to assess VIPN in younger individuals who cannot describe their symptoms are still needed.

While most children experienced only mild to moderate VIPN, others developed severe symptoms. High cumulative vincristine dosage, as well as more frequent dosing, and prolonged treatment duration are well-known predisposing factors for the development of VIPN (Verstappen et al., 2005; Gomber et al., 2010). Other factors influencing neuropathy may include

Table 4. VIPN and pain by cluster ( $n=109$ children).

\begin{tabular}{|c|c|c|c|c|c|c|c|c|c|}
\hline & \multicolumn{3}{|c|}{$\begin{array}{l}\text { Cluster } 1 \text { ( } 187 \\
\text { assessments } \\
\text { from } 14 \text { children) }\end{array}$} & \multicolumn{3}{|c|}{$\begin{array}{c}\text { Cluster } 2 \text { (762 } \\
\text { assessments } \\
\text { from } 70 \text { children) }\end{array}$} & \multicolumn{3}{|c|}{$\begin{array}{c}\text { Cluster } 3 \text { (389 } \\
\text { assessments } \\
\text { from } 25 \text { children) }\end{array}$} \\
\hline & $\mathrm{n}$ & Mean & SD & $\mathrm{n}$ & Mean & SD & $\mathrm{n}$ & Mean & SD \\
\hline \multicolumn{10}{|l|}{ TNS(C-PV items } \\
\hline Paresthesias & 181 & 0.49 & 1.14 & 702 & 0.18 & 0.74 & 376 & 0.06 & 0.40 \\
\hline Numbness & 182 & 0.53 & 1.16 & 700 & 0.09 & 0.54 & 376 & 0.02 & 0.18 \\
\hline Neuropathic pain & 181 & 0.67 & 1.30 & 707 & 0.19 & 0.72 & 376 & 0.09 & 0.51 \\
\hline Temperature & 177 & 0.92 & 1.31 & 622 & 0.30 & 0.76 & 354 & 0.05 & 0.27 \\
\hline Vibration & 173 & 1.51 & 1.46 & 590 & 0.77 & 1.11 & 355 & 0.28 & 0.69 \\
\hline Strength & 175 & 1.25 & 1.15 & 681 & 0.52 & 0.71 & 368 & 0.22 & 0.46 \\
\hline Reflex & 174 & 2.53 & 1.47 & 696 & 1.57 & 1.24 & 359 & 0.91 & 0.99 \\
\hline Autonomic & 185 & 0.84 & 0.91 & 749 & 0.51 & 0.79 & 384 & 0.37 & 0.69 \\
\hline Cranial & 184 & 0.11 & 0.33 & 750 & 0.06 & 0.27 & 384 & 0.04 & 0.22 \\
\hline Total score & 165 & 8.02 & 5.22 & 559 & 4.22 & 3.01 & 342 & 1.94 & 1.86 \\
\hline CTCAE@ motor & 161 & 0.90 & 0.95 & 629 & 0.33 & 0.58 & 343 & 0.11 & 0.32 \\
\hline CTCAE(C) sensory & 161 & 1.03 & 0.89 & 629 & 0.42 & 0.55 & 343 & 0.18 & 0.37 \\
\hline FACES® & 180 & 0.68 & 1.34 & 729 & 0.13 & 0.56 & 377 & 0.06 & 0.41 \\
\hline \multicolumn{10}{|l|}{ NPSC-CIN items } \\
\hline Feet pain & 168 & 0.42 & 1.09 & 601 & 0.06 & 0.34 & 341 & 0.03 & 0.29 \\
\hline Hand pain & 168 & 0.17 & 0.67 & 602 & 0.02 & 0.20 & 341 & 0.01 & 0.10 \\
\hline Jaw pain & 168 & 0.10 & 0.42 & 601 & 0.03 & 0.20 & 341 & 0.02 & 0.27 \\
\hline Numb pain & 168 & 0.42 & 1.03 & 597 & 0.04 & 0.22 & 340 & 0.02 & 0.26 \\
\hline Tingly pain & 168 & 0.29 & 0.90 & 596 & 0.06 & 0.36 & 339 & 0.04 & 0.32 \\
\hline
\end{tabular}

CTCAE $\odot$, Common Terminology Criteria; TNS(C-PV, Total Neuropathy Score-Pediatric Vincristine; VIPN, vincristine-induced peripheral neuropathy. 
Table 5. VIPN and pain by age.

\begin{tabular}{|c|c|c|c|c|c|c|c|c|c|}
\hline & \multicolumn{3}{|c|}{$\begin{array}{l}<5 \text { years* }(630 \\
\text { assessments } \\
\text { from } 53 \text { children) }\end{array}$} & \multicolumn{3}{|c|}{$\begin{array}{l}5-9 \text { years } *(563 \\
\text { assessments } \\
\text { from } 51 \text { children })\end{array}$} & \multicolumn{3}{|c|}{$\begin{array}{l}\geq 10 \text { years } *(346 \\
\text { assessments } \\
\text { from } 37 \text { children })\end{array}$} \\
\hline & $\mathrm{n}$ & Mean & SD & $\mathrm{n}$ & Mean & SD & $\mathrm{n}$ & Mean & SD \\
\hline \multicolumn{10}{|l|}{ TNS(C-PV items } \\
\hline Paresthesias & 434 & 0.10 & 0.46 & 557 & 0.15 & 0.65 & 339 & 0.35 & 1.05 \\
\hline Numbness & 433 & 0.04 & 0.26 & 556 & 0.05 & 0.37 & 340 & 0.38 & 1.03 \\
\hline Neuropathic pain & 440 & 0.21 & 0.66 & 558 & 0.16 & 0.68 & 339 & 0.34 & 1.01 \\
\hline Temperature & 307 & 0.15 & 0.44 & 527 & 0.30 & 0.83 & 336 & 0.49 & 0.98 \\
\hline Vibration & 266 & 0.44 & 0.66 & 523 & 0.59 & 1.05 & 335 & 1.18 & 1.37 \\
\hline Strength & 459 & 0.43 & 0.65 & 538 & 0.48 & 0.68 & 333 & 0.73 & 0.99 \\
\hline Reflex & 496 & 1.31 & 1.21 & 526 & 1.33 & 1.18 & 333 & 1.99 & 1.39 \\
\hline Autonomic & 623 & 0.58 & 0.82 & 555 & 0.50 & 0.78 & 341 & 0.49 & 0.78 \\
\hline Cranial & 624 & 0.10 & 0.37 & 555 & 0.07 & 0.29 & 340 & 0.07 & 0.27 \\
\hline Total score & 228 & 3.29 & 2.35 & 507 & 3.48 & 3.07 & 331 & 5.53 & 4.25 \\
\hline CTCAE@ motor & 543 & 0.24 & 0.52 & 485 & 0.27 & 0.54 & 281 & 0.56 & 0.77 \\
\hline CTCAE $C$ sensory & 543 & 0.31 & 0.53 & 485 & 0.34 & 0.52 & 281 & 0.67 & 0.74 \\
\hline \multicolumn{10}{|l|}{ NPSC-CIN items } \\
\hline Feet pain & 276 & 0.10 & 0.37 & 532 & 0.05 & 0.34 & 333 & 0.20 & 0.79 \\
\hline Hand pain & 275 & 0.03 & 0.19 & 532 & 0.02 & 0.20 & 333 & 0.09 & 0.46 \\
\hline Jaw pain & 274 & 0.02 & 0.12 & 532 & 0.05 & 0.33 & 333 & 0.04 & 0.26 \\
\hline Numb pain & 268 & 0.07 & 0.35 & 531 & 0.02 & 0.18 & 333 & 0.20 & 0.73 \\
\hline Tingly pain & 267 & 0.08 & 0.35 & 530 & 0.06 & 0.38 & 333 & 0.14 & 0.63 \\
\hline
\end{tabular}

CTCAE $\odot$, Common Terminology Criteria; TNS@-PV, Total Neuropathy Score-Pediatric Vincristine; VIPN, vincristine-induced peripheral neuropathy.

${ }^{*}$ Age-based samples are not mutually exclusive because multiple assessments from the same child were obtained over the first year, as the child aged.

hepatic insufficiency, nutritional deficits (Kumar, 2007), and genetics (Argyriou et al., 2012). For example, the CYP3A family of enzymes that metabolizes vinca alkaloids such as vincristine is highly polymorphic, which may result in differential gene expression (Egbelakin et al., 2011). Preliminary evidence suggests that patients who are CYP3A5 high expressers experience less VIPN than those who are CYP3A5 low expressers (Dennison et al., 2006; Egbelakin et al., 2011). Because CYP3A5 is more commonly highly expressed in African Americans compared to Caucasians, Caucasian children as a population may be at higher risk of developing VIPN. We did not observe this association, possibly due to the small number of African American patients in our study population. In a recent study by Diouf et al., another genetic polymorphism was associated with both greater risk of and more severe VIPN (Diouf et al., 2015). Our understanding of the factors that predispose these higher risk children to VIPN is incomplete.

Our results may inform future predictive algorithms to facilitate identification of patients in both the high and low VIPN clusters. For the high VIPN group, this could provide an opportunity to avoid significant, irreversible toxicity in children with low risk ALL by pre-emptively decreasing vincristine dosing, completely eliminating vincristine from ALL therapy, or focusing study of neuroprotective agents in this high-risk population. At the other end of the spectrum, children who are in the low VIPN group but who are identified as having high-risk ALL might benefit from dose intensification with vincristine.

Our findings can be used to inform families about common VIPN signs and symptoms, when they are most likely to occur, and how severe they may be. Families should know that VIPN will likely be an issue throughout the first year of ALL treatment and possibly longer. Because ALL is most prevalent in children 2-3 years of age (American Cancer Society, 2013) who may not be able to report VIPN symptoms, increased knowledge of VIPN will enable everyone - clinicians and families - to anticipate problems in very young children so that symptoms can be reported as early as possible.

Our study provides new information about VIPN patterns, severity, clinical manifestations, and predictors, which are fundamental to identifying approaches to treatment or prevention of VIPN. If severity is associated with specific germline mutations, prospective genetic testing might be used to identify high-risk patients. If drug exposure or other pharmacokinetic parameters is associated with symptom severity, fast metabolizers might tolerate and benefit from higher than current standard vincristine dosing to optimize survival. Development of such a personalized approach will require a valid and reliable approach to quantifying the VIPN phenotype in future studies correlating biomarkers to outcomes. 
Our study has several limitations. First, children who could not cooperate with a full TNS@-PV assessment $(n=19)$ were excluded from our main analysis. Those excluded were younger and more likely to be male. Because older patients develop more severe neuropathy, excluding young patients from the analysis may have resulted in an inflated incidence estimate. We were able to capture deep tendon reflex scores in $79 \%$ of the excluded subset. Reflex scores worsened over time in $50 \%$ of a small subset of the excluded patients (four of eight) who provided more than one score over the first year. These limited findings support our main finding that VIPN is less severe in younger children. In young children, reflex assessment appears to be the most feasible and valid way to assess VIPN, including changes over time.

The cluster analysis results may have been confounded by unknown differences inherent to the enrollment site. Although we assessed for the presence of confounders that might explain this finding, the study was underpowered to detect differences in demographic or other variables by enrollment site. One possible explanation for the higher VIPN scores reported by participants at the George Washington University is that, at this site, a neurologist conducted $20 \%$ of the TNS@ assessments. However, CTCAE@ grades obtained by non-neurologist clinicians at this site, who were blinded to the TNS@-PV scores, were highly correlated with the TNSC scores $(r=0.81 ; p<0.00001)$. It is unlikely that the higher scores obtained at the George Washington University were in any way related to evaluator skill.

Another limitation is that we retained the constipation item within the TNS@-PV, even though our previous psychometric work suggests that the constipation item may be an invalid measure of autonomic neuropathy; many factors influence constipation incidence such as opioid use, diet, hydration, and activity patterns (Smith et al., 2013). However, we retained this item within the TNS@-PV because constipation severity cluster analysis patterns (Fig. 3) mirrored other items, providing some evidence of its validity. Autonomic neuropathy manifesting as constipation, dizziness, or changes in skin temperature is difficult to quantify. Gilchrist and Tanner used the ped-mTNS(C) to quantify autonomic neuropathy by asking patients about dizziness and hot or cold sensations in the extremities and were unable to support the item's validity when comparing scores to those obtained in normal controls (Gilchrist and Tanner, 2013). Autonomic neuropathy may be too difficult to capture and possibly should be eliminated from future pediatric TNS@ versions. Another limitation is that use of analgesic medications such as gabapentin, pregabalin, or narcotics was not quantified and may have impacted pain assessments in patients with VIPN. Last, dose density and cluster analysis interpretation were qualitative in nature.

\section{Acknowledgements}

Special thanks go to R. Case, CCRP, S. Maraldo, CCRP, C. Stephens, A. Stovall, BS, and C. Watson for assisting with data collection and management; A. Angiolillo, MD, W. Jerkens, PNP, and M. S. Lewis, PNP, for assisting with patient recruitment and study coordination; J. Albert, PNP, F. Boyle, BA, A. Egbelakin, MD, MPH, A. Haddix, CPNP, J. P. Kelly, IV, R. Knoerl, BSN, RN, A. Lehmann, BS, C. Mowbray, PNP, PhD, T. Rouse, BS, T. J. Ward, PNP, and M. T. Wood, PNP, for assisting with neuropathy evaluations.

\section{References}

American Cancer Society (2013). Childhood leukemia. http:// www.cancer.org. Updated 2013. Accessed October 25, 2013.

American Cancer Society (2014). Cancer Facts \& Figures 2014. http://www.cancer.org. Accessed February 26, 2015.

Anghelescu DL, Faughnan LG, Jeha S, Relling MV, Hinds PS, Sandlund JT, Cheng C, Pei D, Hankins G, Pauley JL, Pui C (2011). Neuropathic pain during treatment for childhood acute lymphoblastic leukemia. Pediatr Blood Cancer 57:1147-1153.

Argyriou AA, Bruna J, Marmiroli P, Cavaletti G (2012). Chemotherapy-induced peripheral neurotoxicity (CIPN): an update. Crit Rev Oncol Hematol 82:51-77.

Bisogno G, Ferrari A, Bergeron C, Scagnellato A, Prete A, Alaggio R, Casanova M, D'Angelo P, Di Cataldo A, Carli M (2005). The IVADo regimen - a pilot study with ifosfamide, vincristine, actinomycin D, and doxorubicin in children with metastatic soft tissue sarcoma: a pilot study of behalf of the European Pediatric Soft Tissue Sarcoma Study Grou. Cancer 103:1719-1724.

Bosenberg A, Thomas J, Lopez T, Kokinsky E, Larsson LE (2003). Validation of a six-graded faces scale for evaluation of postoperative pain in children. Paediatr Anaesth 13:708-713.

Cavaletti G, Frigeni B, Lanzani F, Mattavelli L, Susani E, Alberti P, Cortinovis D, Bidoli P (2010). Chemotherapy-induced peripheral neurotoxicity assessment: a critical revision of the currently available tools. Eur J Cancer 46:479-494.

Cavaletti G, Cornblath DR, Merkies IS, Postma TJ, Rossi E, Frigeni $B$, Alberti $P$, Bruna J, Velasco R, Argyriou AA, Kalofonos HP, Psimaras D, Ricard D, Pace A, Galie E, Briani C, Dalla Torre C, Faber CG, Lalisang RI, Boogerd W, Brandsma D, Koeppen S, Hense J, Storey D, Kerrigan S, Schenone A, Fabbri S, Valsecchi MG, Cl-PeriNomS Group (2013). The chemotherapy-induced peripheral neuropathy outcome measures standardization study: from consensus to the first validity and reliability findings. Ann Oncol 24:454-462.

Chauvenet AR, Shashi V, Selsky C, Morgan E, Kurtzberg J, Bell B, Pediatric Oncology Group S (2003). Vincristine-induced neuropathy as the initial presentation of Charcot-Marie-Tooth disease in acute lymphoblastic leukemia: a Pediatric Oncology Group study. J Pediatr Hematol Oncol 25:316-320.

Cornblath DR, Chaudhry V, Carter K, Lee D, Seysedadr M, Miernicki M, Joh T (1999). Total neuropathy score: validation and reliability study. Neurology 53:1660-1664. 
Dennison JB, Kulanthaivel P, Barbuch RJ, Renbarger JL, Ehlhardt WJ, Hall SD (2006). Selective metabolism of vincristine in vitro by CYP3A5. Drug Metab Dispos 34:1317-1327.

Diouf B, Crews KR, Lew G, Pei D, Cheng C, Bao J, Zheng JJ, Yang W, Fan Y, Wheeler HE, Wing C, Delaney SM, Komatsu M, Paugh SW, McCorkle JR, Lu X, Winick NJ, Carroll WL, Loh ML, Hunger SP, Devidas M, Pui CH, Dolan ME, Relling MV, Evans WE (2015). Association of an inherited genetic variant with vincristine-related peripheral neuropathy in children with acute lymphoblastic leukemia. JAMA 313:815-823.

Egbelakin A, Ferguson M, MacGill E, Lehmann A, Topletz A, Quinney S, Li L, McCammack K, Hall S, Renbarger J (2011). Increased risk of vincristine neurotoxicity associated with low CYP3A5 expression genotype in children with acute lymphoblastic leukemia. Pediatr Blood Cancer 56:361-367.

Frigeni B, Piatti M, Lanzani F, Alberti P, Villa P, Zanna C, Ceracchi M, Ildebrando M, Cavaletti G (2011). Chemotherapy-induced peripheral neurotoxicity can be misdiagnosed by the National Cancer Institute Common Toxicity Scale. J Peripher Nerv Syst 16:228-236.

Gilchrist L (2012). Chemotherapy-induced peripheral neuropathy in pediatric cancer patients. Semin Pediatr Neurol 19:9-17.

Gilchrist LS, Tanner L (2013). The pediatric-modified Total Neuropathy Score: a reliable and valid measure of chemotherapy-induced peripheral neuropathy in children with non-CNS cancers. Support Care Cancer 21:847-856.

Gilchrist LS, Marais L, Tanner L (2014). Comparison of two chemotherapy-induced peripheral neuropathy measurement approaches in children. Support Care Cancer 22:359-366.

Gomber S, Dewan P, Chhonker D (2010). Vincristine induced neurotoxicity in cancer patients. Indian J Pediatr 77:97-100.

Griffith K, Merkies ISJ, Hill E, Cornblath D (2010). Measures of chemotherapy-induced peripheral neuropathy: a systematic review of psychometric properties. J Peripher Nerv Syst 15:314-325.

Hockenberry MJ (Ed) (2005). Wong's Essentials of Pediatric Nursing, 7th Edn. Elsevier Mosby, St. Louis, pp 1307.

Hoffman MC, Mulrooney DA, Steinberger J, Lee J, Baker KS, Ness KK (2013). Deficits in physical function among young childhood cancer survivors. J Clin Oncol 31:2799-2805.

Kaufman L, Rousseeuw P (1987). Clustering by Means of Medoids, Vol. 3. Faculty of Mathematics and Informatics, Delft University of Technology.

Khan RB, Hudson MM, Ledet DS, Morris EB, Pui CH, Howard SC, Krull KR, Hinds PS, Crom D, Browne E, Zhu L, Rai S, Srivastava D, Ness KK (2014). Neurologic morbidity and quality of life in survivors of childhood acute lymphoblastic leukemia: a prospective cross-sectional study. J Cancer Surviv 8:688-96.

Kortmann RD, Kuhl J, Timmermann B, Mittler U, Urban C, Budach V, Richter E, Willich N, Flentje M, Berthold F, Slavc I, Wolff J, Meisner C, Wiestler O, Sorensen N, WarmuthMetz M, Bamberg M (2000). Postoperative neoadjuvant chemotherapy before radiotherapy as compared to immediate radiotherapy followed by maintenance chemotherapy in the treatment of medulloblastoma in childhood: results of the German prospective randomized trial HIT '91. Int J Radiat Oncol Biol Phys 46:269-279.

Kumar N (2007). Nutritional neuropathies. Neurol Clin 25:209-255
Liew E, Thyagu S, Atenafu EG, Alibhai SM, Brandwein JM (2013). Quality of life following completion of treatment for adult acute lymphoblastic leukemia with a pediatric-based protocol. Leuk Res 37:1632-1635.

Messinger $Y$, Gaynon P, Raetz E, Hutchinson R, Dubois $S$, Glade-Bender J, Sposto R, van der Giessen J, Eckroth E, Bostrom BC (2010). Phase I study of bortezomib combined with chemotherapy in children with relapsed childhood acute lymphoblastic leukemia (ALL): a report from the Therapeutic Advances in Childhood Leukemia (TACL) Consortium. Pediatr Blood Cancer 55:254-259.

Messinger YH, Gaynon PS, Sposto R, van der Giessen J, Eckroth E, Malvar J, Bostrom BC, Therapeutic Advances in Childhood Leukemia \& Lymphoma (TACL) Consortium (2012). Bortezomib with chemotherapy is highly active in advanced B-precursor acute lymphoblastic leukemia: Therapeutic Advances in Childhood Leukemia \& Lymphoma (TACL) study. Blood 120:285-290.

Ness KK, Jones KE, Smith WA, Spunt SL, Wilson CL, Armstrong GT, Srivastava DK, Robison LL, Hudson MM, Gurney JG (2013). Chemotherapy-related neuropathic symptoms and functional impairment in adult survivors of extracranial solid tumors of childhood: results from the St Jude Lifetime Cohort study. Arch Phys Med Rehabil 94:1451-1457.

Paice JA (2009). Clinical challenges: chemotherapy-induced peripheral neuropathy. Semin Oncol Nurs 25:S8-S19.

Postma TJ, Benard BA, Huijgens PC, Ossenkoppele GJ, Heimans JJ (1993). Long-term effects of vincristine on the peripheral nervous system. J Neurooncol 15:23-27.

Postma TJ, Heimans JJ, Muller MJ, Ossenkoppele GJ, Vermorken JB, Aaronson NK (1998). Pitfalls in grading severity of chemotherapy-induced peripheral neuropathy. Ann Oncol 9:739-744.

Ramchandren S, Leonard M, Mody RJ, Donohue JE, Moyer J, Hutchinson R, Gurney JG (2009). Peripheral neuropathy in survivors of childhood acute lymphoblastic leukemia. J Peripher Nerv Syst 14:184-189.

Smith EL, Beck SL, Cohen J (2008). The total neuropathy score (TNS): a tool for measuring chemotherapy-induced peripheral neuropathy. Oncol Nurs Forum 35:96-102.

Smith EM, Li L, Hutchinson RJ, Ho R, Burnette WB, Wells E, Bridges C, Renbarger J (2013). Measuring vincristine-induced peripheral neuropathy in children with acute lymphoblastic leukemia. Cancer Nurs 6:E49-60.

Toopchizadeh V, Barzegar M, Rezamand A, Feiz AH (2009). Electrophysiological consequences of vincristine contained chemotherapy in children: a cohort study. J Pediatr Neurol 7:351-356.

Vats T, Buchanan G, Mehta P, Ragab A, Hvizdale E, Nitschke R, Link M, Beardsley GP, Maybee D, Krischer J (1992). A study of toxicity and comparative therapeutic efficacy of vindesine-prednisone vs. vincristine-prednisone in children with acute lymphoblastic leukemia in relapse. A Pediatric Oncology Group study. Invest New Drugs 10:231-234.

Verstappen CC, Koeppen S, Heimans JJ, Huijgens PC, Scheulen ME, Strumberg D, Kiburg B, Postma TJ (2005). Dose-related vincristine-induced peripheral neuropathy with unexpected off-therapy worsening. Neurology 64:1076-1077. 